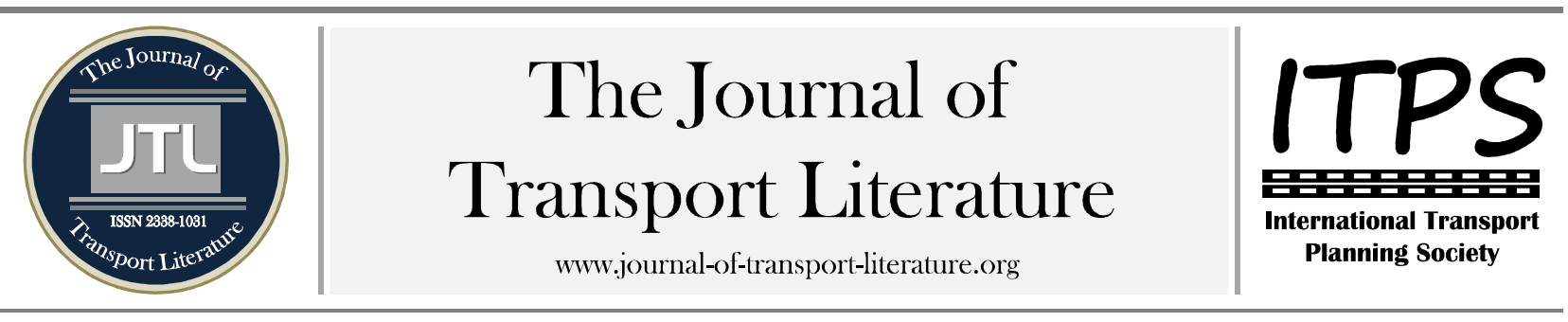

\title{
Proposta de um modelo para a avaliação da segurança operacional em aeroportos baseado nos métodos Borda e Promethee II
}

Tiago José Menezes Gonçalves+; Anderson Ribeiro Correia

Aeronautics Institute of Technology, São José dos Campos, Brazil

\section{Article Info}

Palavras-chave:

aeroportos

indicadores de segurança

multicritério

Promethee

Borda

Submitted 7 Apr 2014;

received in revised form 2 Sep 2014;

accepted 9 Oct 2015.

Licensed under

Creative Commons

CC-BY 3.0 BR.

\section{Resumo}

\begin{abstract}
Este trabalho propõe uma abordagem para a obtenção de um índice global de segurança operacional em pistas aeroportuárias, através da análise de eventos de segurança. Nela ponderam-se os eventos de forma proporcional aos riscos à segurança deles resultantes. A partir da classificação de risco de cada evento, utiliza-se o método Borda para a obtenção de pesos e o método Promethee II para o cálculo de um índice global de segurança. Esta abordagem foi aplicada no Aeroporto Internacional de São Paulo/Guarulhos. Os resultados indicam quedas similares de 3,5\% em relação à média no nível de segurança global nos anos 3 e 4. No período mais recente os desempenhos unicriteriais foram positivos em 7 dos 10 critérios avaliados, apresentando queda de 37\%, 41\% e 17\% em relação à média nos indicadores de colisões de aeronaves, colisões de propriedades e danos causados por objetos estranhos respectivamente. Por fim, foram recomendadas ações para elevar os níveis de segurança do sistema aeroportuário. A ponderação com o método Borda foi considerada consistente e o índice global, obtido com o método Promethee II, foi considerado representativo do nível de segurança atingido no sistema aeroportuário.
\end{abstract}

+ Corresponding author. Instituto Tecnológico de Aeronáutica. Praça Marechal Eduardo Gomes, 50. 12280250 - São José dos Campos, SP - Brasil.

E-mail address: tiagojmg@ita.br.

\section{Introdução}

Este trabalho tem como objetivo propor uma abordagem para a obtenção de um índice global de segurança para pistas aeroportuárias. Os métodos Borda e Promethee II são utilizados de forma a calcular tal índice com base em eventos de segurança operacional (ESO). Adicionalmente, este trabalho apresenta os seguintes objetivos específicos: (i) ponderar os critérios avaliados para a obtenção do índice global de forma proporcional ao risco à segurança por eles causado, com base em sua classificação de risco; (ii) realizar uma análise do desempenho global da segurança aeroportuária de forma a identificar tendências e diagnosticar problemas; e (iii) realizar recomendações para apoiar o planejamento de ações para a elevação dos níveis de segurança aeroportuária.

0 problema de obter um índice de segurança global em pistas aeroportuárias foi analisado primeiramente por Lobianco e Correia (2013). Eles propuseram uma metodologia empírica baseada na realização de uma média ponderada do número de ocorrências de cada tipo de ESO ocorridos em determinado período, arbitrando-se os pesos com base na matriz de classificação de riscos definida pela ICAO (2009). A insuficiência de bases teóricas deste estudo revelou a necessidade de uma nova abordagem com o uso de modelos capazes de fornecer resultados mais representativos da realidade, cujos cálculos pudessem ser defendidos diante de um decisor.

Na busca por uma base teórica, Gonçalves, Correia e Lobianco (2014) propuseram a utilização da Multi-Attribute Value Theory - MAVT para a obtenção do índice global de segurança, fundamentando-o nas teorias de apoio multicritério à decisão. A MAVT não utiliza pesos, mas taxas de substituição, que foram calculadas utilizando-se o método Rank Order Centroid - ROC (Ahn, 2011) com base nas três classes de riscos (inaceitáveis, aceitáveis desde que sejam mitigados e aceitáveis) definidas na matriz de classificação adotada pela ICAO (2009). 0 uso destas três classes forneceu três valores para representar as taxas de substituição de todos os critérios, o que possibilitou uma diferenciação pequena entre as taxas, concluindo-se que o cálculo de taxas de substituição com este procedimento retorna somente três valores possíveis para representar as taxas de todos os critérios, o que foi considerado insuficiente para descrever de forma completa toda a diferença entre critérios analisados.

Destaca-se que tanto a média ponderada utilizada por Lobianco e Correia (2013) quanto a MAVT utilizada por Gonçalves, Correia e Lobianco (2014) são métodos compensatórios, nos quais o alto desempenho em um critério pode compensar um desempenho baixo em outro e, portanto, não refletir este aspecto no índice global de segurança.

Neste contexto, este trabalho propõe um modelo para contornar as limitações citadas previamente, propondo uma integração do método Borda com o método Promethee II. 0 método Borda é utilizado de forma a ponderar as classificações da matriz de risco conforme o procedimento proposto por Garvey e Lansdowne (1997). Adicionalmente, o método Promethee II é utilizado para o cálculo de um índice global de segurança, cuja agregação dos valores de desempenho dos critérios é não compensatória. 
Como resultados, o método Borda possibilitou agrupar as classificações de risco dos ESO em nove categorias de ponderação, concluindo-se que sua aperfeiçoou os resultados obtidos empiricamente por Lobianco e Correia (2013), os quais obtiveram valores sem base conceitual e considerados superdimensionados; e por Gonçalves, Correia e Lobianco (2014), que utilizaram o método ROC e obtiveram somente três valores nos quais se agrupavam todas as classificações de risco. Além disso, a abordagem foi utilizada para analisar a segurança no Aeroporto Internacional de São Paulo/Guarulhos, indicando uma redução do nível de segurança de 3,5\% em relação à média na transição do ano 2 para o 3, permanecendo no mesmo patamar no ano 4 e subindo $+1 \%$ em relação à média no ano 5 . Em relação aos desempenhos unicriteriais, no período mais recente eles foram positivos no período mais recente em 7 dos 10 critérios avaliados, apresentando queda de $37 \%, 41 \%$ e $17 \%$ em relação à média nos indicadores de colisões de aeronaves, colisões de propriedades e danos causados por objetos estranhos respectivamente. Esta análise possibilitou o planejamento de ações para a elevação dos níveis de segurança nos critérios com baixo desempenho.

\section{Segurança e Riscos Aeroportuários}

Diversos trabalhos existentes na literatura abordaram o tema de avaliação da segurança e/ou riscos aeroportuários utilizando diferentes perspectivas, dentre eles Horowitz e Santos (2009), Fu e Chan (2014) e Pacheco, Fernandes e Domingos (2014).

De acordo com Roelen e Klompstra (2012), o desempenho de um Sistema de Gerenciamento de Segurança Operacional (SGSO) aeroportuário é monitorado por meio de indicadores de desempenho de segurança. Tradicionalmente, taxas de acidentes eram utilizadas para avaliar o desempenho em segurança, porém uma maior base de dados estatísticos tornou-se necessária quando a segurança aumentou e os acidentes tornaram-se raros, dificultando o procedimento de avaliação.

De forma a obter uma maior base de dados para a realização de análises, os dados de ESO constituem uma alternativa às taxas de acidentes, possibilitando a utilização de análises mais refinadas ao inserir incidentes e demais eventos de menores proporções na análise e proporcionar um maior histórico de dados.

Para a avaliação do desempenho da segurança em pistas aeroportuárias é necessário que os ESO utilizados como indicadores de desempenho sejam ponderados, de forma que aqueles que apresentem maior risco de comprometer a segurança sejam monitorados com maior rigor (Lobianco e Correia, 2013). Para a classificação de riscos a ICAO (2009) utiliza a matriz apresentada na Figura 1.

\begin{tabular}{|c|c|c|c|c|c|}
\hline \multirow[b]{2}{*}{$\begin{array}{c}\text { Probabilidade d } \\
\text { ocorrência }\end{array}$} & \multicolumn{5}{|c|}{ Severidade do risco } \\
\hline & $\begin{array}{c}\text { Catastrófico } \\
\text { A }\end{array}$ & $\begin{array}{c}\text { Crítico } \\
\text { B }\end{array}$ & $\begin{array}{c}\text { Significativo } \\
\mathrm{C}\end{array}$ & $\begin{array}{c}\text { Pequeno } \\
\text { D }\end{array}$ & $\begin{array}{c}\text { Insignificante } \\
\mathrm{E}\end{array}$ \\
\hline Frequente & $5 \mathrm{~A}$ & $5 \mathrm{~B}$ & $5 \mathrm{C}$ & $5 \mathrm{D}$ & $5 \mathrm{E}$ \\
\hline Ocasional & $4 \mathrm{~A}$ & $4 B$ & $4 \mathrm{C}$ & $4 \mathrm{D}$ & $4 \mathrm{E}$ \\
\hline Remoto & $3 \mathrm{~A}$ & $3 B$ & $3 \mathrm{C}$ & $3 \mathrm{D}$ & $3 \mathrm{E}$ \\
\hline Improvável & $2 \mathrm{~A}$ & $2 B$ & $2 \mathrm{C}$ & $2 \mathrm{D}$ & $2 \mathrm{E}$ \\
\hline $\begin{array}{l}\text { Extremamente } \\
\text { improvável }\end{array}$ & $1 \mathrm{~A}$ & $1 \mathrm{~B}$ & $1 \mathrm{C}$ & 1D & $1 \mathrm{E}$ \\
\hline
\end{tabular}

Figura 1 - Matriz de classificação de riscos. Fonte: ICAO (2009).

Nesta matriz, o risco de um ESO é classificado em função de dois critérios: a probabilidade de ocorrência e sua severidade. Os riscos correspondentes às células $5 \mathrm{~A}, 5 \mathrm{~B}, 5 \mathrm{C}, 4 \mathrm{~A}, 4 \mathrm{~B}$ e $3 \mathrm{~A}$ são classificados como inaceitáveis sob as circunstâncias existentes, os riscos 5D, 5E, 4C, 4D, 4E, 3B, 3C, 3D, 2A, 2B e 2C como aceitáveis desde que sejam mitigados e os demais riscos são classificados como aceitáveis. Nas seções seguintes estas classes são denominadas respectivamente como C1, C2 e C3.

\section{Métodos Borda e Promethee}

O método Borda foi utilizado neste trabalho por possibilitar pontuar e ordenar alternativas quando somente estão disponíveis informações ordinais sobre o desempenho dos critérios analisados. Além disso, ele é um dos métodos multicriteriais com procedimentos de cálculo mais simples, resultando em maior transparência para os decisores. Conforme Emerson (2011) o método Borda foi inicialmente proposto como um método de votação, onde havendo $\mathrm{N}$ candidatos e múltiplos eleitores, cada eleitor atribuía $\mathrm{N}-1$ pontos ao candidato considerado como o melhor, N-2 pontos ao segundo melhor, e assim por diante. A pontuação de cada candidato era somada e o vencedor era aquele que obtinha a maior pontuação. Além disso, este método pode ser utilizado para apoiar decisões multicriteriais, onde os decisores ordenam as alternativas em relação a cada critério e posteriormente somam seus pontos, sendo preferível a alternativa com maior pontuação. Neste sentido, se rik é a ordenação da alternativa i no critério k, a pontuação bi da alternativa i no método Borda é obtida conforme apresentado na Equação (1).

$$
b i=\sum_{k}(N-r i k)
$$

Dentre as aplicações apresentadas na literatura que fizeram uso do método Borda no setor aeroespacial e na área de avaliação de riscos, destacam-se Garvey e Lansdowne (1997) e Fernandes et al. (2013). Em relação ao método Promethee, ele pertence à família dos métodos de sobreclassificação. Esses métodos visam construir uma relação de sobreclassificação para representar as preferências dos decisores e resolver a problemática de ordenação (Gonçalves e Belderrain, 2012).

Este método foi utilizado neste trabalho por ser um método não compensatório, em que o desempenho baixo em um critério não é compensado indefinidamente por desempenhos altos nos demais. Considerando que em problemas de avaliação da segurança um único critério com baixo desempenho pode tornar todo o sistema inseguro, um método não compensatório consiste em uma opção mais adequada. Os procedimentos para a utilização deste método estão amplamente documentados na literatura, podendo ser conferidos em Ishizaka e Nemery (2013). Uma revisão completa de literatura sobre o método Promethee pode ser encontrada em Behzadian et al. (2010). 


\section{Metodologia}

A Figura 2 apresenta o modelo proposto neste trabalho para calcular um índice global e avaliar a segurança de pistas aeroportuárias com base em ESO. Neste modelo, os diferentes tipos de ESO registrados no SGSO aeroportuário são utilizados para definir os critérios para a obtenção de um índice global de segurança em pistas aeroportuárias.

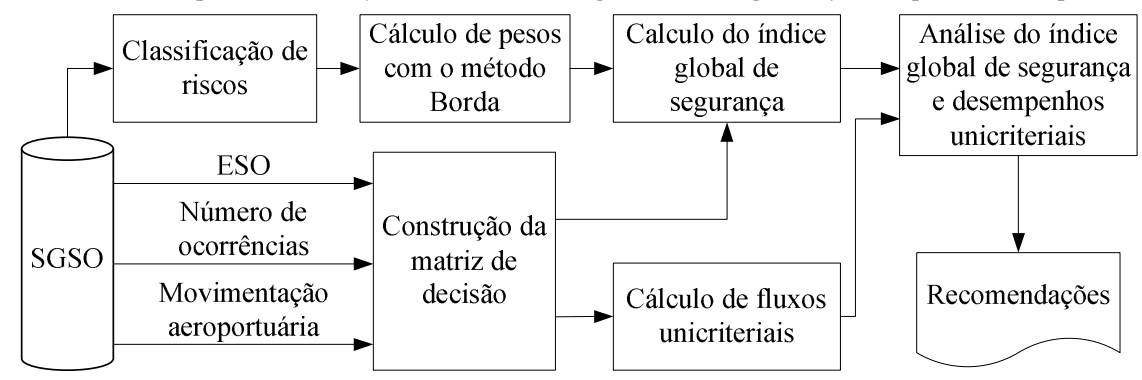

Figura 2 - Modelo proposto. Fonte: Elaborado pelos autores.

Na primeira etapa, uma matriz de decisão $\mathrm{D}=($ qij$) \mathrm{m} \times \mathrm{n}$ é construída a partir do histórico de ESO. Devido a alterações ocorridas na movimentação aeroportuária ao longo do tempo, Lobianco e Correia (2013) afirmam que deve se inserir na matriz de decisão o número de ESO ocorridos a cada 100.000 aeronaves movimentadas, calculado conforme a Equação (2).

$$
q i j=\frac{100.000 \times Q i j}{m i}
$$

Na Equação 2, qij representa número de ocorrências do evento j no ano i para cada 100 mil aeronaves movimentadas; Qij o número de ocorrências do evento j no ano i; e mi consiste no número de aeronaves movimentadas no ano i. Paralelamente à construção da matriz de decisão, as classificações de risco apresentadas na Figura 1 são ponderadas de forma proporcional ao risco. Uma vez que a cada critério corresponde um ESO, o peso atribuído à classificação de risco deste evento é utilizado como o ponderador do respectivo critério. Este trabalho utiliza a abordagem proposta por Garvey e Lansdowne (1997), que realiza uma análise bicriterial baseada no método Borda. Esta abordagem utiliza como critérios as informações ordinais presentes na matriz de classificação de riscos referentes à probabilidade de ocorrência e severidade do risco, pontuando-os com valores que variam de zero a quatro e somando-os para encontrar a peso de cada classificação de risco. Considerando dois tipos de ESO cujos riscos sejam classificados como 5A e 2C, eles seriam ponderados com os valores 16 e 3 respectivamente. Finalizando, as classificações de risco são obtidas do SGSO e cada critério (correspondente a um ESO) é ponderado com o método Borda. Uma vez construída a matriz de decisão e obtidos os pesos, prossegue-se para o cálculo do índice global de segurança com a utilização do método Promethee II. Neste sentido, a contribuição deste trabalho consiste em integrar os métodos Borda e Promethee II, de forma a desenvolver um modelo para o cálculo de um índice global de segurança que supere as limitações das abordagens citadas anteriormente. Por fim, o índice global de segurança (representado pelo fluxo líquido) e os fluxos unicriteriais de cada ano são analisados de forma a realizar recomendações para o aperfeiçoamento da segurança aeroportuária.

\section{Aplicação}

\subsection{Objeto de estudo}

O Aeroporto Internacional de São Paulo/Guarulhos foi escolhido como objeto de estudo para exemplificar a aplicação da abordagem proposta neste trabalho, sendo escolhido por apresentar a maior movimentação de aeronaves e passageiros no Brasil.

\subsection{Definição de critérios}

A definição dos critérios teve como base o trabalho de Lobianco e Correia (2013), sendo o foco deste trabalho a análise dos ESO ocorridos em pistas aeroportuárias. Além disso, a escolha dos ESO foi embasada na disponibilidade de informações acessíveis sobre número de ocorrências de cada evento, sendo eles:

Colisão de aeronave no pátio (c1): refere-se a uma colisão no pátio envolvendo uma ou mais aeronaves sem intenção de voo. São contabilizadas neste indicador as colisões entre uma aeronave e um veículo ou entre uma aeronave e um equipamento qualquer.

Colisão entre aeronave e ave (c2): consiste em uma colisão de uma aeronave com uma ou mais aves, ocorrida em pistas aeroportuárias quando uma aeronave estiver realizando os procedimentos de decolagem, pouso ou taxi.

Colisão entre propriedades (c3): refere-se a uma colisão que envolva veículos, equipamentos ou outras propriedades. Este indicador contabiliza colisões entre: (i) um veículo ou equipamento móvel com uma edificação; (ii) um veículo ou equipamento móvel com um objeto qualquer; (iii) dois veículos ou equipamentos em movimento; (iv) um veículo ou equipamento em movimento com um veículo ou equipamento estacionado; e (v) um veículo ou equipamento em movimento com um veículo ou equipamento parado.

Objeto estranho na área de movimento (c4): este indicador contabiliza o número de objetos estranhos encontrados em área de movimento. São exemplos dessa ocorrência a presença de metais, plásticos, tecidos, isopor, gelo, pavimento, papéis, ferramentas, madeira, balões, pipas, etc. trator.

Desprendimento de reboque (c5): consiste em um desprendimento de equipamento rebocável (dolly) de seu veículo

Estacionamento fora da área de segurança (c6): este indicador contabiliza o número de ocorrências em que um equipamento ou veículo são deixados fora da área de segurança, bem como o número de aeronaves estacionadas fora de sua posição de estacionamento.

Incursão em pista (c7): ocorre quando há a presença não autorizada de aeronave, veículo ou pessoa em uma superfície designada para pouso ou decolagem de uma aeronave. 
Ingresso de animal não voador (c8): refere-se à entrada de um animal não voador em área de movimento. Estre esses animais estão capivaras, cachorros, jacarés, cobras, etc.

Lesão corporal (c9): refere-se a uma lesão provocada por um acidente de trabalho no pátio, por um acidente com passageiro ou por um atropelamento.

Vazamento (c10): consiste na ocorrência de um derramamento de fluido proveniente de uma aeronave, veículo ou outro equipamento. São exemplos de fluidos derramados os óleos hidráulicos, óleos lubrificantes, óleo diesel, combustíveis, etc.

Depois de definidos quais ESO seriam utilizados como critérios, prosseguiu-se para a ponderação dos critérios.

\subsection{Ponderação dos critérios}

Os pesos dos critérios foram calculados de forma proporcional ao risco à segurança aeroportuária oferecida pelo ESO correspondente. Essa ponderação foi realizada com base na classificação de riscos proposta pela ICAO, a qual é utilizada pelo SGSO aeroportuário. Considerando que esta classificação utiliza dados ordinais na definição da probabilidade e severidade de cada ESO, o método Borda foi utilizado para gerar as ponderações de cada tipo de evento.

A probabilidade e severidade foram utilizadas como critérios, sendo pontuada cada classificação apresentada na Figura 1 utilizando-se a Equação (1). Cada pontuação consistiu na soma das pontuações obtidas no critério de probabilidade com a pontuação obtida no critério de severidade, conforme o algoritmo do método Borda. As classificações de risco que obtiveram pontuações de mesmo valor foram agrupadas. Considerando que os critérios de c1 a c10 apresentam as classificações de risco 4C, 3D, 4C, 2D, 4C, 2E, 2A, 3E, 3C e 5D no SGSO aeroportuário, utilizando as informações da Tabela 1, estes critérios foram ponderados utilizando os pesos $5,3,5,2,5,1,5,2,4$ e 5 respectivamente.

\begin{tabular}{cc|cc}
\multicolumn{4}{c}{ Tabela 1 - Ponderação por classificação de risco. Fonte: Elaborada pelos autores. } \\
\hline Classificações de Risco & Pontuação & Classificações de Risco & Pontuação \\
\hline $5 \mathrm{~A}$ & 8 & $4 \mathrm{E}, 3 \mathrm{D}, 2 \mathrm{C}, 1 \mathrm{~B}$ & 3 \\
$5 \mathrm{~B}, 4 \mathrm{~A}$ & 7 & 3E, 1C, 2D & 2 \\
$5 \mathrm{C}, 4 \mathrm{~B}, 3 \mathrm{~A}$ & 6 & 2E, 1D & 1 \\
$5 \mathrm{D}, 4 \mathrm{C}, 3 \mathrm{~B}, 2 \mathrm{~A}$ & 5 & $1 \mathrm{E}$ & 0 \\
$5 \mathrm{E}, 4 \mathrm{D}, 3 \mathrm{C}, 2 \mathrm{~B}, 1 \mathrm{~A}$ & 4 & - & - \\
\hline
\end{tabular}

\subsection{Avaliação de desempenho}

Para a obtenção de um índice global de segurança foi utilizado o método Promethee II e os dados da matriz de decisão contendo o número de ocorrências (por ano) dos ESO que correspondem a cada critério. Neste cálculo foi utilizada uma função de preferência de "forma V", com limiar de indiferença igual à zero para todos os critérios e as amplitudes dos desempenhos foram utilizadas para representar os limiares de preferência. A Tabela 2 apresenta os fluxos líquidos unicriteriais e globais $(\phi)$ relativos ao desempenho da segurança operacional em pistas aeroportuárias em cada ano.

Tabela 2 - Fluxos líquidos globais e unicriteriais. Fonte: Elaborada pelos autores.

\begin{tabular}{c|cccccccccc|c}
\hline Período & $c 1$ & c2 & c3 & c4 & c5 & c6 & c7 & c8 & c9 & c10 & $\phi$ \\
\hline Ano 1 & 0,51 & $-0,29$ & 0,25 & $-0,30$ & $-0,25$ & $-0,74$ & $-0,04$ & $-0,83$ & 0,44 & $-0,78$ & $-0,10$ \\
Ano 2 & 0,36 & 0,66 & 0,43 & 0,66 & $-0,25$ & $-0,09$ & $-0,78$ & 0,13 & 0,62 & 0,47 & 0,22 \\
Ano 3 & 0,05 & $-0,04$ & 0,30 & 0,58 & $-0,59$ & 0,03 & $-0,08$ & 0,38 & $-0,63$ & $-0,06$ & $-0,07$ \\
Ano 4 & $-0,17$ & $-0,59$ & $-0,15$ & $-0,59$ & 0,43 & 0,28 & 0,43 & $-0,11$ & $-0,59$ & 0,10 & $-0,07$ \\
Ano 5 & $-0,74$ & 0,26 & $-0,82$ & $-0,34$ & 0,66 & 0,51 & 0,47 & 0,42 & 0,15 & 0,28 & 0,02 \\
\hline
\end{tabular}

Segundo a análise dos fluxos líquidos globais, a segurança em pistas aeroportuárias obteve melhor desempenho no ano 2 , seguido respectivamente dos anos 5, 4, 3 e 1. Esta análise indica que o desempenho global não manteve uma tendência de melhoria contínua, evidenciada pelas quedas de desempenho similares de 3,5\% em relação à média ocorridas nos anos 3 e 4. Considerando-se o ano 5 como o mais representativo do estado atual do sistema, os critérios com menores fluxos líquidos unicriteriais foram priorizados para a realização de ações para mitigação de riscos. A análise dos fluxos líquidos indica que o nível de segurança operacional em pistas aeroportuárias apresentou no último período uma melhoria de desempenho de $1 \%$ em relação à média. Embora a segurança tenha aumentado ao considerar todo o sistema, os critérios c1, c3 e c4 sofreram queda de respectivamente $37 \%$, 41\% e 37\% em relação a média.

\subsection{Recomendações}

De forma a atuar no planejamento e melhoria do desempenho dos critérios c1, c3 e c4 priorizados na seção anterior, foram realizadas recomendações à administração aeroportuária sobre quais ações de mitigação realizar. Em relação ao critério c1 (colisões entre aeronaves), foi recomendada a adoção das seguintes providências: realização de inspeção/revisão anual (técnica e documental) de equipamentos e veículos que ingressam nos pátios e pistas, desenvolvimento de políticas de inspeções rotineiras pelos fiscais de pátio, reforço da sinalização (ex.: cones refletivos) para demarcar os limites extremos da área de estacionamento de cada aeronave, realização de treinamentos enfatizando a importância de ter atenção em relação à movimentação de veículos e equipamentos próximos a aeronaves, realização de treinamentos sobre as normas operacionais do pátio e a realização de manutenção preventiva de veículos e equipamentos. Quanto ao critério c3 (colisões com propriedades), observou-se que a maioria das colisões registradas foi causada por falta de atenção de seus condutores, pelo descumprimento de normas, por falhas de equipamentos e de procedimentos. Como resultado, em adição às providências recomendadas para o critério $\mathrm{c} 1$, as seguintes ações foram recomendadas: realização de cursos de atualização de condutores envolvidos em ocorrências, emissão de alertas específicos postados em locais de grande circulação e por o controle do movimento de veículos por meio de tags eletrônicos semelhantes aos utilizados em pedágios. No caso do critério c4 (danos causados por objetos estranhos), ele refere-se principalmente a incidentes/acidentes ocorridos quando objetos são ingeridos por motores ou colidem contra aeronaves. Buscando melhorar o desempenho neste indicador, foram propostas as seguintes ações: aumentar a frequência com que são realizadas fiscalizações em pistas e taxiways para a retirada de objetos estranhos, promover campanhas de conscientização sobre as consequências da presença de objetos estranhos para toda a comunidade aeroportuária e atuar junto à empresas terceirizadas para que estas reavaliem seus procedimentos de forma a aumentar a segurança operacional do aeroporto. 


\title{
Conclusão
}

Neste trabalho, o procedimento proposto para a ponderação de critérios possibilitou a obtenção de um maior conjunto de pesos e uma base conceitual nas teorias do Apoio Multicritério à Decisão, fornecendo maior consistência e acurácia ao processo de modelagem e contribuindo para contornar as limitações dos trabalhos previamente realizados sobre este tema. Ressalta-se também que o cálculo dos pesos de forma proporcional ao risco possibilitou fornecer maior ênfase aos critérios que impactam mais fortemente na segurança operacional, minimizando distorções nos resultados devido à supervalorização de critérios de pouca influência na segurança. A utilização do método Borda na ponderação dos critérios foi considerada simples e consistente, possibilitando o cálculo de pesos de forma transparente para a gestão aeroportuária. Em relação ao método Promethee II, sua característica não compensatória foi considerada adequada aos aspectos do problema abordado, visto que desempenhos muito baixos em um critério não são acobertados por desempenhos altos nos demais critérios. Além disso, este método utiliza comparações pareadas onde as alternativas que apresentam melhores desempenhos na maioria dos critérios obtêm pontuações positivas para o fluxo líquido. Como consequência, a obtenção de fluxos líquidos positivos em determinado período significa que ele obteve um desempenho melhor que o desempenho médio dos demais períodos, o que torna fácil a interpretação dos resultados.

Como trabalhos futuros sugere-se a realização de modelagens utilizando funções de preferência não lineares, de forma a verificar se existe alguma vantagem em sua utilização quando comparada as funções de preferência utilizadas neste trabalho. Sugere-se investigar a aplicabilidade de outros métodos multicriteriais, comparando-os com os resultados obtidos neste trabalho, assim como outros métodos de ponderação, de forma a verificar alternativas para a obtenção de pesos em aeroportos/aeródromos onde a classificação de riscos não é confiável ou mesmo inexistente. Por fim, ressalta-se que a aplicação do modelo proposto foi considerada eficiente pelos administradores aeroportuários, colaborando para a continuidade de sua aplicação prática como parte integrante no controle e melhoria de desempenho operacional do SGSO aeroportuário.

\section{Agradecimentos}

Os autores agradecem ao Conselho Nacional de Desenvolvimento Científico e Tecnológico (CNPq) pelo apoio prestado a realização deste trabalho.

\section{Referências}

Ahn, B. S. (2011). Compatible weighting method with rank order centroid: Maximum entropy ordered weighted averaging approach. European Journal of Operational Research, 212(3), 552-559. doi:10.1016/j.ejor.2011.02.017

Behzadian, M., Kazemzadeh, R. B., Albadvi, A., \& Aghdasi, M. (2010). Promethee: A comprehensive literature review on methodologies and applications. European Journal of Operational Research, 200, 198-215. doi:10.1016/j.ejor.2009.01.021

Emerson, P. (2011). The original Borda count and partial voting. Social Ch and Welfare, 40(2), 353-358. doi:10.1007/s00355-011-0603-9

Fernandes, J. E. de M., Gomes, L. F. A. M., Mello, J. C. C. B. S. de, \& Júnior, S. F. G. (2013). Seleção de uma aeronave para atividades de charter regional com utilização do método multicritério de borda modificado com utilização de mediana. Journal of Transport Literature, $7(2), 171-191$.

Fu, Y. K., \& Chan, T. L. (2014). A conceptual evaluation framework for organisational safety culture: An empirical study of Taipei Songshan Airport. Journal of Air Transport Management, 34, 101-108. doi:10.1016/j.jairtraman.2013.08.005

Garvey, P. R., \& Lansdowne, Z. F. (1997). Risk matrix an approach for identifying, assessing, and ranking program risks. Air Force Journal of Logistics, 22(1), 18-23.

Gonçalves, T. J. M., Correia, A. R., \& Lobianco, J. F. B. (2014). Multi-criteria approach to evaluate airport safety level. In Air Transport Research Society (Ed.), Air Transport Research Society World Conference (pp. 1-12). Bordeaux: Air Transport Research Society.

Gonçalves, T. J. M., \& Belderrain, M. C. N. (2012). Performance evaluation with Promethee GDSS and GAIA: A study on the ITA-SAT satellite project. Journal of Aerospace Technology and Management, 4(3), 381-392. doi:10.5028/jatm.2012.04033411

Horowitz, B. M., \& Santos, J. R. (2009). Runway safety at airports: A systematic approach for implementing ultra-safe options. Journal of Air Transport Management, 15(6), 357-362. doi:10.1016/j.jairtraman.2009.04.006

ICAO. (2009). Doc 9859 - Safety Management Manual (SMM). (ICAO, Ed.) (2nd ed., pp. 1-284). Montréal: ICAO.

Ishizaka, A., \& Nemery, P. (2013). Multi-criteria decision analysis: Methods and software (pp. 1-296). Chichester: Wiley.

Lobianco, J. F. B., \& Correia, A. R. (2013). Methodology to obtain airport safety indicators using Safety Management Systems. Journal of the Brazilian Air Transportation Research Society, 9(1), 47-58.

Pacheco, R. R., Fernandes, E., \& Domingos, E. M. (2014). Airport airside safety index. Journal of Air Transport Management, 34, 86-92. doi:10.1016/j.jairtraman.2013.08.007

Roelen, A. L. C., \& Klompstra, M. B. (2012). The challenges in defining aviation safety performance indicators. In 11th International Probabilistic Safety Assessment and Management Conference and the Annual European Safety and Reliability Conference 2012 (pp. 25-29). Helsink: Curran Associates. Retrieved from www.proceedings.com/16286.html.

\begin{abstract}
This study developed a model for the evaluation, control and improvement of airport safety by analyzing operational incidents. Considering the multidimensional feature of this evaluation, a model based on multicriteria Borda and Promethee methods was proposed. The operational incidents were used in the development of criteria and the weighting of each of them was carried out in proportion to the respective safety risk. Moreover, its application was performed at an airport in order to identify trends, diagnose problems and support action planning and provide a basis for allocating resources for prevention and mitigation of safety hazards. This application was used to analyze the validity of the model, and conclusions about their efficiency and applicability were obtained.
\end{abstract}

Key words: airports, safety indicator, multicriteria, Promethee, Borda. 